\title{
Epileptogenic Lesion Quantification in MRI Using Contralateral 3D Texture Comparisons
}

\author{
Oscar Alfonso Jiménez del Toro ${ }^{1}$, Antonio Foncubierta-Rodríguez ${ }^{1}$, \\ María Isabel Vargas Gómez ${ }^{2}$, Henning Müller ${ }^{1,2}$, and Adrien Depeursinge ${ }^{1,2}$ \\ 1 University of Applied Sciences Western Switzerland (HES-SO) \\ ${ }^{2}$ University and University Hospitals of Geneva, Switzerland
}

\begin{abstract}
Epilepsy is a disorder of the brain that can lead to acute crisis and temporary loss of brain functions. Surgery is used to remove focal lesions that remain resistant to treatment. An accurate localization of epileptogenic lesions has a strong influence on the outcome of epilepsy surgery. Magnetic resonance imaging (MRI) is clinically used for lesion detection and treatment planning, mainly through simple visual analysis. However, visual inspection in MRI can be highly subjective and subtle 3D structural abnormalities are not always entirely removed during surgery. In this paper, we introduce a lesion abnormality score based on computerized comparison of the 3D texture properties between brain hemispheres in T1 MRI. Overlapping cubic texture blocks extracted from user-defined 3D regions of interest (ROI) are expressed in terms of energies of 3D steerable Riesz wavelets. The abnormality score is defined as the Hausdorff distance between the ROI and its corresponding contralateral region in the brain, both expressed as ensembles of blocks in the feature space. A classification based on the proposed score allowed an accuracy of $85 \%$ with 10 control subjects and 8 patients with epileptogenic lesions. The approach therefore constitutes a valuable tool for the objective pre-surgical evaluation of patients undergoing epilepsy surgery.
\end{abstract}

Keywords: 3D texture, Riesz transform, epilepsy, CAD.

\section{Introduction}

Brain alterations on the cortical mantle are often associated with neurological disorders such as epilepsy [18]. A precise localization of the epileptogenic lesions in the brain is an important step in selecting the appropriate treatment or intervention for these patients 16. The most common way of visualizing the lesions is to use multi-planar rendering in 3D magnetic resonance imaging (MRI) [13. When lesions can be visualized in MRI clearly, epilepsy surgery is associated with better outcome [19. However, alterations of the brain tissue are often subtle and differentiating them from healthy tissue is a difficult task 2011]. Studies regarding lesion detection by visual examination of MRI in patients undergoing surgery have reported an accuracy of $50-60 \%$ at 1.5 Tesla [16]17.

MRI post-processing methods have been proposed to enhance visualization for certain types of highly epileptogenic lesions such as focal cortical dysplasia 
(FCD) [1 2411. In some cases, these methods have shown to outperform visual assessment in discerning subtle anatomical changes, particularly in formerly cryptogenic epilepsy where focal lesions were not detected by visual assessment of MRI 413. In [111, voxel-based morphometry was used to create feature maps based on the characteristics of FCD visible on MRI. Other approaches also show high accuracy for lesion detection using gray matter quantification or anatomical feature analysis like the depth of the brain sulci 4 4 . These methods still require analysis and interpretation of the results by an experienced radiologist and their sensitivity depends on subjective visual confirmation and personal experience [922]. The created feature maps highlight a suspicious region of interest (ROI) that is then analyzed in the original MRI. Methods using voxel-based morphometry require the use of templates or atlases to normalize the anatomical variability of a patient's brain anatomy. This normalization affects the local characteristics of the tissue texture in sometimes already subtle lesions [7,11/23]. In other methods [4] that use visual features based on gray-level co-occurrence matrices (GLCM), the number of gray-levels are often reduced to compute a reliable and simple representation of the texture properties 21. GLCMs require arbitrary choices of scales, orientations and an exhaustive exploration of these parameters is needed for an accurate characterization of the subtle texture properties of epileptogenic lesions. This is not appropriate in three-dimensions since the number of possible orientations grows exponentially compared to 2D. 3D Riesz wavelet transforms yield multiscale and multi-orientation analysis of local biomedical tissue properties [6 8 ] and are expected to enable the characterization of subtle alterations of the brain tissue.

The question addressed in this paper is: Does texture analysis using 3D Riesz wavelet transforms on previously obtained ROIs allow an objective measurement of candidate epileptogenic lesions when compared to healthy brain tissue? An abnormality score based on comparisons of the cortical brain tissue texture between a ROI with candidate epileptogenic lesion and the corresponding contralateral healthy anatomical region is proposed. The abnormality score from subtle epileptogenic lesions can help clinicians in quantitatively and objectively assess 3D brain texture properties. This information is crucial in the pre-surgical planning of patients with epilepsy for a better classification of the lesion and eventually improve the outcome of the surgical resection.

\section{Materials and Methods}

Dataset: The database used for this article consists of eight cases with seizures caused by a lesion located in the brain and 10 control cases without visible anomalies. The 8 patients with epilepsy underwent surgery and had confirmed diagnosis of the proposed epileptogenic lesion. These lesions include 5 cases with dysplasia, 1 ganglioglioma, 1 cavernoma and 1 case with tuberous sclerosis (see Table 1). All cases have complete MRI series including T1 weighted, T2-Fluid Attenuated Inversion Recovery (FLAIR) images and diffusion tensor imaging (DTI) as part of an epilepsy pre-surgical standard control. They were acquired 
on a $3 \mathrm{~T}$ MRI scanner (Magnetom Trio, a Tim System, Siemens, Germany) using a head coil. The high resolution isotropic T1-weighted 3D dataset was obtained consisting of sagittal and coronal planes $(\mathrm{TR}=1.6-1.9 \mathrm{~ms}, \mathrm{TE}=2.32 \mathrm{~ms}$, flip angle $=9$ ) and were re-sliced as axial slices for a better visualization of the lesions. The images have a matrix of dimensions $192 \times 255 \times 512$, with a voxel size $=$ $0.9 \mathrm{~mm} \times 0.9 \mathrm{~mm} \times 0.449 \mathrm{~mm}$ in the axial view. Epileptogenic lesions were manually delineated by a neuroradiologist for each case in 3D. Annotations were made on the MR image where the lesion was most visible (i.e., either T1, T2-FLAIR or DTI). In 4 patients the lesion was not clearly visible in T1 weighted MR. When the manual annotation was made on the T2-FLAIR or DTI image of the patient, the coordinate transformation was estimated using image registration for the T1 weighted image of the patient. T1 weighted images have good tissue delineation and are best for discriminating gray matter [13]. The T1 weighted images are then transformed to Neuroimaging Informatics Technology Initiative (NIfTI) format and bias-corrected for intensity inhomogeneities with smooth basis functions using statistical parametric mapping (SPM 1 ) to reduce the effect of the spatially varying artifact that modulates the intensity of MRI [11. The smoothing was applied evenly to each patient and has limited effect on texture comparisons.

Table 1. Overview of the epileptogenic lesions

\begin{tabular}{lccccc}
\hline & age & lesion type & lobe & MRI annotation & Volume $\left(\mathrm{cm}^{3}\right)$ \\
\hline Patient 1 & 19 & Dysplasia & Parietal & DTI axial & 5.53 \\
Patient 2 & 18 & Dysplasia & Frontal & T2-FLAIR axial & 0.51 \\
Patient 3 & 49 & Cavernoma & Temporal & T2-FLAIR axial & 0.18 \\
Patient 4 & 22 & Tuberous Sclerosis & Occipital & T2-FLAIR axial & 0.17 \\
Patient 5 & 17 & Ganglioglioma & Temporal T2-FLAIR sagittal & 0.27 \\
Patient 6 & 21 & Dysplasia & Parietal & T2-FLAIR axial & 23.1 \\
Patient 7 & 20 & Dysplasia & Frontal & T2-FLAIR axial & 12.35 \\
Patient 8 & 13 & Dysplasia & Parietal & T2-LAIR coronal & 11.51 \\
\hline
\end{tabular}

3D Texture Analysis Based on Directional Riesz Wavelets: 3D Riesz filterbanks are used to characterize the texture properties of the brain cortex in MRI. 3D Riesz wavelets are steerable and multiscale and yield overcomplete characterization of local scales and orientation properties [68. They are therefore able to model subtle local 3D texture properties with high reproducibility compared to other methods. The latter require arbitrary selection of scales and orientations, and the probability that they match actual texture properties of small epileptogenic lesions is low [1]. The $N$-th order Riesz transform $\boldsymbol{\mathcal { R }}^{(N)}$ of a threedimensional signal $f(\boldsymbol{x})$ is defined in the Fourier domain as:

$$
\widehat{\mathcal{R}^{\left(n_{1}, n_{2}, n_{3}\right)}} f(\boldsymbol{\omega})=\sqrt{\frac{n_{1}+n_{2}+n_{3}}{n_{1} ! n_{2} ! n_{3} !}} \frac{\left(-j \omega_{1}\right)^{n_{1}}\left(-j \omega_{2}\right)^{n_{2}}\left(-j \omega_{3}\right)^{n_{3}}}{\|\boldsymbol{\omega}\|^{n_{1}+n_{2}+n_{3}}} \hat{f}(\boldsymbol{\omega}),
$$

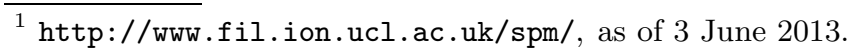


I. Manual ROI annotation

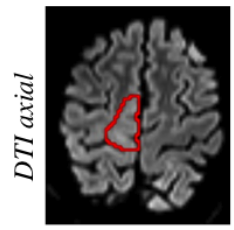

III. Intermodality registration

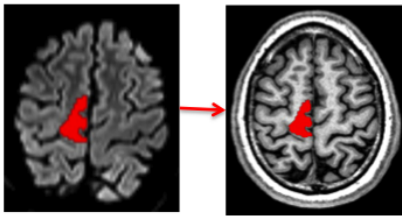

V. Inter-patient registration

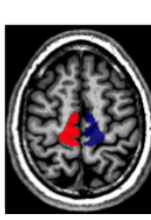

Patient

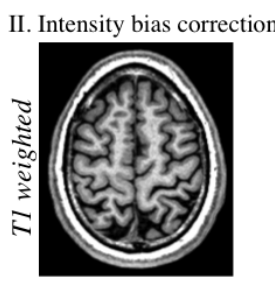

IV. Contralateral hemisphere

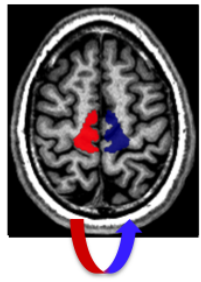

VI. Texture analysis

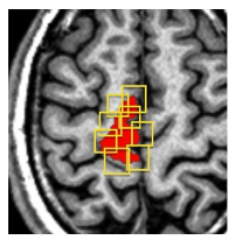

Control set

Fig. 1. Pipeline of the method; the implemented texture analysis of suspicious ROIs with the description of each step is included in the experimental setup subsection

for all combinations of $\left(n_{1}, n_{2}, n_{3}\right)$ with $n_{1}+n_{2}+n_{3}=N$ and $n_{1,2,3} \in \mathbb{N}$. Eq. (1) yields $\left(\begin{array}{c}N+2 \\ 2\end{array}\right)$ templates $\mathcal{R}^{\left(n_{1}, n_{2}, n_{3}\right)}$ and forms multiscale steerable filterbanks when coupled with a multi-resolution framework based on isotropic band-limited wavelets (e.g., Simoncelli) [6]. It therefore allows continuous descriptions of three-dimensional scales and orientations.

Experimental Setup: The manual annotations were used as ROIs to perform 3D Riesz wavelet texture analysis since it is common practice to estimate the pathological origin of the seizures during pre-surgical planning [16]. A contralateral comparison of the cortical brain texture in the ROI to the opposite hemisphere's same area of the patient is performed. This is a clinical approach commonly used in the visual evaluation of the lesions when physicians want to compare the same anatomical region of interest to the lesion-free contralateral brain hemisphere. A similar approach for brain texture analysis was used in 310.

To obtain the ROIs in the contralateral anatomical region, the hemispheres are registered using the Elastix software 2 [15]. It uses affine registration that

${ }^{2}$ http://elastix.isi.uu.nl, as of 3 June 2013. 
globally aligns both hemispheres using an iterative stochastic gradient descent optimizer with a multi-resolution approach [14. The obtained ROIs were all visually inspected to ensure that they label the corresponding anatomical region in the opposite brain hemisphere of the patient. For each patient, both the candidate lesion and the contralateral ROI are registered with the same registration method to the complete control set for testing 3D Riesz wavelet analysis on the cortical tissue. The $\mathrm{T} 1$ images of the patients with epilepsy are set as moving images and registered independently to the T1 fixed images of the control set using affine registration. In the end, one ROI is found for each hemisphere for each patient with epilepsy in each of the 10 control patients. This yields 80 inter-hemisphere texture analysis comparisons with 160 brain ROIs based on the manual annotations made by the neuroradiologist.

The images are preprocessed to have an isotropic voxel resolution, which is obtained by dividing samples along the $z$ axis. ROIs are divided into $32^{3}$ overlapping blocks, where the location of the block centers are randomly chosen inside the region. Varying numbers of 100, 200 and 500 blocks were used for the validation. Whenever the number of samples was larger than the total number of voxels in the ROI, the instances were repeated to make sure that all patients had the same number of blocks tested for texture analysis. Based on previous work [8], we found that $N=2$ and 4 scales (i.e., 24 subbands) provided a good trade-off between the dimensionality of the feature space and the wealth of the filterbanks. The energy of the Riesz coefficients of the 24 subbands were used for each block.

The Hausdorff distance (HD) 12 was used as a distance measure between the subsets of blocks for each ROI and its contralateral anatomical region with a Euclidean metric in the feature space composed by the energies of coefficients of each 24 Riesz subbands. Given two finite point sets $A=\left\{a_{1}, \ldots, a_{p}\right\}$ and $B=\left\{b_{1}, \ldots, b_{q}\right\}, \mathrm{HD}$ is defined as:

$$
H(A, B)=\max (h(A, B), h(B, A))
$$

where

$$
h(A, B)=\max _{\mathbf{a} \in \mathbf{A}} \min _{\mathbf{b} \in \mathbf{B}}\|a-b\|_{l_{2}} .
$$

High HD reflects a high texture difference between the two subsets of coefficients. The proposed method is unsupervised and requires no training or optimization.

\section{Results}

The mean HD of the 10 control patients' inter-hemisphere comparison in each registered ROI of 8 epilepsy patients is compared to the mean HD of the interhemisphere comparison of 8 patients with epileptogenic lesions. Fig. 2 shows the mean of the HD from contra lateral comparisons for each patient and the corresponding anatomical region in the control set. A lesion is classified as positive or negative based on the comparison of the HD values of the control set. An accuracy of $85 \%$, specificity of $90 \%$, positive predictive value of $89.47 \%$ and negative 


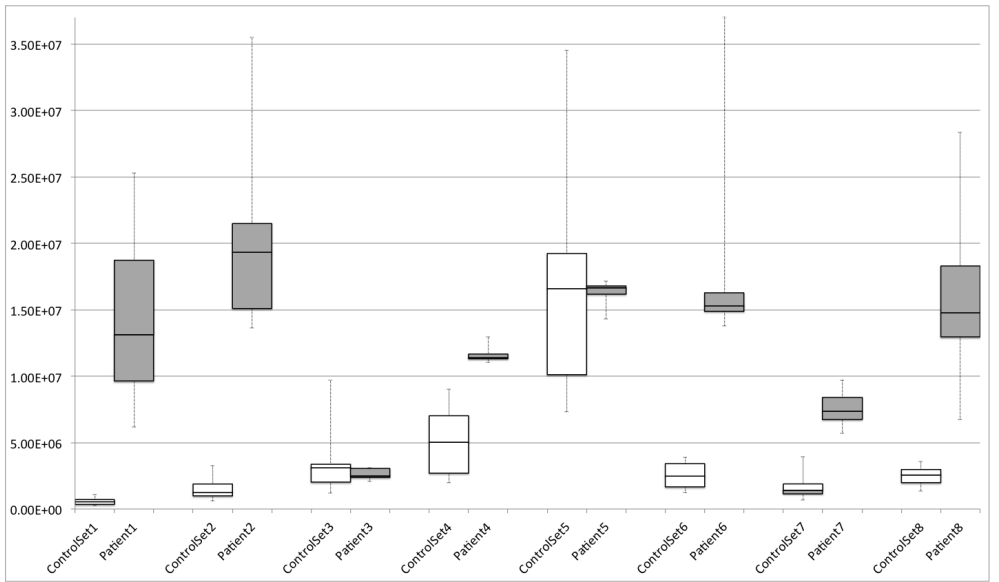

Fig. 2. Box-plot showing the comparisons of HD between the patient ROI and the same region in the control set. The graph shows the mean HD box-plot of the control set when the inter-hemisphere comparison is applied to the same ROI as the one with the patient. Side-by-side visualization of the results shows the effectiveness of the method for 6 out of 8 patients to detect a higher inter-hemisphere HD in the patients with epilepsy. This shows that the epileptogenic lesion modifies local texture properties of most anatomical regions in the dataset.

predictive value $85.71 \%$ are obtained for the 80 comparisons ( 8 patients $\times 10$ control cases) in 160 ROIs based on manual annotations of a neuroradiologist.

\section{Discussions and Conclusions}

We propose a method for 3D texture analysis of cortical brain tissue with candidate epileptogenic lesions to be used in patients undergoing surgery for drugresistant epilepsy. The method provides a quantitative difference measure between the texture in an ROI to the contra lateral healthy anatomical region in the brain. This measure can be used in clinical routine to objectively quantify abnormal tissue and consequently assist in the decision making on treatment of patients with subtle epileptogenic lesions. The results show that the Hausdorff distance between subsets of the energies of Riesz wavelet coefficients can discriminate between ROIs in control cases and patients with a suspicious abnormality. There are 4 patients in the dataset where the lesions are not detected in the $\mathrm{T} 1$ sequence by visual examination alone. This strengthens our view that the proposed approach can be valuable for both detection and quantification of these lesions for surgery preparation. The epileptogenic lesion in patient 5 is located at the most anterior part of the temporal lobe. This is a site where there are more variations from the left and right hemispheres, even for normal cases. All abnormality scores are therefore higher in this region. The challenge in performing the method without inter-patient normalization is to overcome 
the influence of anatomical variability and inter-hemisphere differences when measuring the candidate lesions. The continuous characterization of scales and orientations possible with Riesz wavelets allows adequate modelling of 3D texture alterations that can be caused by epileptogenic lesions.

An objective of future work is to provide a lesion segmentation based on the inter-hemisphere comparison of the abnormality score in inter-patient ROIs using voxel-by-voxel difference of the 3D Riesz wavelet coefficients from various samples inside the ROI. The patients will benefit from better interpretation of the available images to improve the outcome of the surgical procedure. 3D texture analysis on T2-FLAIR and DTI will be included to fuse the information from all pre-surgical standard control MRI series and improve the robustness and specificity of our method. Enlargement of our database is also foreseen.

Acknowledgments. This work was supported by the EU/FP7 through VISCERAL (318068), Khresmoi (257528) and the SNSF (grant 205320-141300/1).

\section{References}

1. Antel, S.B., Collins, D.L., Bernasconi, N., Andermann, F., Shinghal, R., Kearney, R.E., Arnold, D.L., Bernasconi, A.: Automated detection of focal cortical dysplasia lesions using computational models of their MRI characteristics and texture analysis. NeuroImage 19(4), 1748-1759 (2003)

2. Bastos, A., Corneau, R.M., Andermann, F., Melanson, D., Cendes, F., Dubeau, F., Fontaine, S., Tampieri, D., Olivier, A.: Diagnosis of subtle focal dysplastic lesions: curvilinear reformatting from three-dimensional magnetic resonance imaging. Annals of Neurology 46, 88-94 (1999)

3. Bergo, F.P.G., Falcão, A.X., Yasuda, C.L., Cendes, F.: FCD segmentation using texture asymmetry of MR-T1 images of the brain. In: 5th IEEE International Symposium on Biomedical Imaging, pp. 424-427 (2008)

4. Bernasconi, A., Bernasconi, N.: Unveiling epileptogenic lessions: The contribution of image processing. Epilepsia 52, 20-24 (2011)

5. Besson, P., Andermann, F., Dubeau, F., Bernasconi, A.: Small focal cortical dysplasia lesions are located at the bottom of a deep sulcus. Brain 131(12), 3246-3255 (2008)

6. Chenouard, N., Unser, M.: 3D steerable wavelets and monogenic analysis for bioimaging. In: 2011 IEEE International Symposium on Biomedical Imaging: From Nano to Macro, pp. 2132-2135 (April 2011)

7. Colliot, O., Bernasconi, N., Khalili, N., Antel, S.B., Naessens, V.B., Bernasconi, A.: Individual voxel-based analysis of gray matter in focal cortical dysplasia. Neuroimage 29(1), 162-171 (2006)

8. Depeursinge, A., Foncubierta-Rodríguez, A., Vargas, A., Van De Ville, D., Platon, A., Poletti, P.A., Müller, H.: Rotation-covariant texture analysis of 4D dual-energy $\mathrm{CT}$ as an indicator of local pulmonary perfusion. In: IEEE 10th International Symposium on Biomedical Imaging, ISBI 2013, pp. 149-152. IEEE (April 2013)

9. Focke, N.K., Yogarajah, M., Symms, M.R., Gruber, O., Paulus, W., Duncan, J.S.: Automated MR image classification in temporal lobe epilepsy. Neuroimage 59(1), 356-362 (2012) 
10. Geremia, E., Clatz, O., Menze, B.H., Konukoglu, E., Criminisi, A., Ayache, N.: Spatial decision forests for ms lesion segmentation in multi-channel magnetic resonance images. NeuroImage 57, 378-390 (2011)

11. Huppertz, H.J., Grimm, C., Fauser, S., Kassubek, J., Mader, I., Hochmuth, A., Spreer, J., Schulze-Bonhage, A.: Enhanced visualization of blurred gray-white matter junctions in focal cortical dysplasia by voxel-based 3D MRI analysis. Epilepsy Research 67(1), 35-50 (2005)

12. Huttenlocher, D.P., Klanderman, G.A., Rucklidge, W.J.: Comparing images using the Hausdorff distance. IEEE Transactions on Pattern Analysis and Machine Intelligence 15(9), 850-863 (1993)

13. Kassner, A., Thornhill, R.E.: Texture analysis: A review of neurologic MR imaging applications. American Journal of Neuroradiology 31, 809-816 (2010)

14. Klein, S., Pluim, J.P., Staring, M., Viergever, M.A.: Adaptive stochastic gradient descent optimisation for image registration. International Journal of Computer Vision 81(3), 227-239 (2009)

15. Klein, S., Staring, M., Murphy, K., Viergever, M.A., Pluim, J.P.: Elastix: a toolbox for intensity-based medical image registration. IEEE Transactions on Medical Imaging 29(1), 196-205 (2010)

16. Mellerio, C., Labeyrie, M.A., Chassoux, F., Daumas-Duport, C., Landré, E., Turak, B., Roux, F.X., Meder, J.F., Devaux, B., Oppenheim, C.: Optimizing MR imaging detection of type 2 focal cortical dysplasia: Best criteria for clinical practice. American Journal of Neuroradiology 33(10), 1932-1938 (2012)

17. Montenegro, M.A., Min Li, L., Guerreiro, M.M., Guerreiro, C.A.M., Fernando, C.: Focal cortical dysplasia: Improving diagnosis and localization with magnetic resonance imaging multiplanar and curvilinear reconstruction. Journal of Neuroimaging 12, 224-230 (2002)

18. Palmini, A., Gambardella, A., Andermann, F., Dubeau, F., da Costa, J.C., Olivier, A., Tampieri, D., Gloor, P., Quesney, F., Andermann, E., Paglioli, E., PaglioliNeto, E., Andermann, L.C., Leblanc, R., Kim, H.I.K.: Intrinsic epileptogenicity of human dysplastic cortex as suggested by corticography and surgical results. Annals of Neurology 37, 476-487 (1995)

19. Riney, C.J., Chong, W.K., Clark, C.A., Cross, J.H.: Voxel based morphometry of FLAIR MRI in children with intractable focal epilepsy: Implications for surgical intervention. European Journal of Radiology 81, 1299-1305 (2012)

20. Tassi, L., Colombo, N., Garbelli, C.R., Francione, S., Lo Russo, G., Mai, R., Cardinale, F., Cossu, M., Ferrario, A., Galli, C., Bramerio, M., Citterio, A., Spreafico, R.: Focal cortical dysplasia: neuropathological subtypes, EEG, neuroimaging and surgical outcome. Brain 125(8), 1719-1732 (2002)

21. Unser, M.: Sum and difference histograms for texture classification. IEEE Transactions on Pattern Analysis and Machine Intelligence 8(1), 118-125 (1986)

22. Wagner, J., Weber, B., Urbach, H., Elger, C.E., Huppertz, H.J.: Morphometric MRI analysis improves detection of focal cortical dysplasia type II. Brain 134, 2844-2854 (2011)

23. Woermann, F.G., Free, S.L., Koepp, M.J., Ashburner, J., Duncan, J.S.: Voxelby-voxel comparison of automatically segmented cerebral gray matter - a raterindependent comparison of structural MRI in patients with epilepsy. Neuroimage 10(4), 373-384 (1999) 